\title{
Transgenic ramie [Boehmeria nivea (L.) Gaud.]: factors affecting the efficiency of Agrobacterium tumefaciens-mediated transformation and regeneration
}

Bo Wang $\cdot$ Lijun Liu $\cdot$ Xuxia Wang •

Jinyu Yang - Zhenxia Sun · Na Zhang •

Shimei Gao $\cdot$ Xiulong Xing $\cdot$ Dingxiang Peng

Published online: 23 September 2009

(C) Springer-Verlag 2009

This article has been retracted because part of the data shown has already been published before, by different authors.

The online version of the original article can be found under doi:10.1007/s00299-009-0732-0.

B. Wang $\cdot$ L. Liu $\cdot$ X. Wang $\cdot$ J. Yang $\cdot$ Z. Sun $\cdot$ N. Zhang

S. Gao $\cdot$ X. Xing $\cdot$ D. Peng $(\bowtie)$

College of Plant Science and Technology,

Huazhong Agricultural University,

430070 Wuhan, Hubei, China

e-mail: wangbo@mail.hzau.edu.cn 